\section{Uma comparação dos custos do transplante renal em relação às diálises no Brasil}

\author{
Cost comparison of kidney transplant versus \\ dialysis in Brazil
}

\begin{abstract}
Una comparación de los costes del trasplante renal en relación con las diálisis en Brasil
\end{abstract}

\begin{abstract}
Resumo
O objetivo do presente estudo foi comparar os custos médicos diretos do transplante renal e das terapias renais substitutivas, especificamente a hemodiálise e a diálise peritoneal, sob a perspectiva do Sistema Único de Saúde (SUS). Os custos das terapias renais substitutivas foram extraídos de informações publicadas na literatura. Os itens de custo previstos do transplante renal foram identificados em um hospital privado mediante coleta dos códigos dos procedimentos utilizados para a cobrança do SUS e os demais itens extraídos da literatura. O resultado desta pesquisa indica que, no período dos quatro anos coberto por este estudo, o transplante renal de doador falecido gera uma economia, por paciente, de $R \$ 37$ mil e $R \$ 74 \mathrm{mil}$ em relação à hemodiálise e à diálise peritoneal, respectivamente. Quanto ao transplante renal de doador vivo, as economias são ainda maiores: $R \$$ 46 mil e $R \$ 82$ mil em relação à hemodiálise e à diálise peritoneal, respectivamente. Este resultado, aliado a análises de sobrevida e qualidade de vida, pode caracterizar o transplante renal como a melhor alternativa do ponto de vista financeiro e clínico, auxiliando na formulação de políticas públicas relacionadas com os transplantes de órgãos no Brasil.
\end{abstract}

Transplante de Rim; Custos e Análise de Custo; Economia
Silvia Brand Silva 1

Heitor Mansur Caulliraux 1

Claudia Affonso Silva Araújo 1

Eduardo Rocha 1

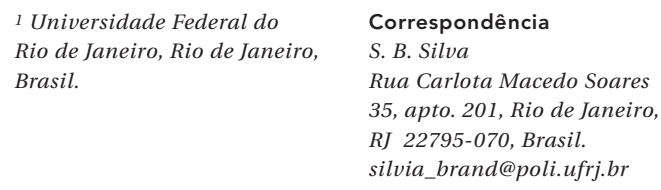

1 Universidade Federal do Rio de Janeiro, Rio de Janeiro, Brasil.

Correspondência

S. B. Silva

Rua Carlota Macedo Soares

35, apto. 201, Rio de Janeiro,

RJ 22795-070, Brasil.

silvia_brand@poli.ufrj.br 


\section{Introdução}

O aumento significativo de pacientes com insuficiência renal crônica representa um sério problema de saúde pública no Brasil por afetar a vida de milhares de brasileiros e onerar os cofres públicos 1 . Na fase terminal da doença, a sobrevivência do paciente é condicionada à realização de um tipo de terapia renal substitutiva - hemodiálise ou diálise peritoneal - ou à realização do transplante renal.

As terapias renais substitutivas são, no Brasil, o tratamento dominante, apesar de gerarem impactos negativos na vida dos pacientes como a perda de quantidade e qualidade de vida ${ }^{2}$. Godoy et al. 3 estimam redução de $11 \%$ na renda de portadores de insuficiência renal crônica por conta da redução da capacidade laboral que poderia, em grande parte, ser atenuada pela realização do transplante 4 .

De acordo com o censo da Sociedade Brasileira de Nefrologia (SBN), a cada ano cerca de 20 mil brasileiros iniciam algum tipo de terapias renais substitutivas. Entre 2000 e 2013, o número de pacientes em diálise em todo o país passou de 42 mil para 100 mil, o que representou um aumento de $138 \% 5$. Esse aumento está associado a mudanças nos padrões de consumo, alteração no estilo de vida e transição demográfica 6 .

Ao analisar a fonte de financiamento, verifica-se que o Sistema Único de Saúde (SUS) arca com grande parte do custo do tratamento de pacientes dialíticos. Em 2012, o SUS financiou $84 \%$ dos pacientes em tratamento em alguma modalidade de terapia renal substitutiva 5 , despendendo 2 bilhões de Reais (todos os procedimentos ambulatoriais que envolvem hemodiálise e diálise peritoneal) (Departamento de Informática do SUS. http://tabnet.data sus.gov.br/cgi/deftohtm.exe?sia/cnv/qauf.def, acessado em 25/Abr/2015). O SUS é também o principal financiador de transplantes no Brasil. O país possui um dos maiores programas públicos de transplantes de órgãos e tecidos do mundo, administrado pelo Sistema Nacional de Transplantes (Sistema Nacional de Transplantes. Gastos com tratamento renal, 2012. http://portalsaude.saude.gov.br/portalsaude/ index.cfm?portal=pagina.visualizarArea $\& \operatorname{cod} A$ rea $=414 \&$ area $=$ transplantes, acessado em 15/ Set/2015) 7. Em 2012, mais de 90\% dos transplantes foram custeados pelo SUS, equivalente a um gasto de 139,6 milhões de Reais (http:// portalsaude.saude.gov.br/index.php/o-minis terio/principal/secretarias/368-sas-raiz/dahu-raiz/transplantes-raiz/transplantes/l2-trans plantes/13304-instituicoes-e-recursos, acessado em 15/Set/2015).
Os gastos iniciais com os procedimentos de transplante renal são elevados devido ao procedimento cirúrgico, que representa um expressivo percentual dos custos. No entanto, os gastos posteriores à cirurgia são relativamente pequenos, quando comparados aos gastos das condutas pré-cirúrgicas e cirúrgicas, e devem-se aos procedimentos de acompanhamento e aos medicamentos que evitam a rejeição do órgão (imunossupressores) ${ }^{8}$. As terapias renais substitutivas, por sua vez, representam gastos anuais levemente ascendentes, já que a perda da função renal é irreversível e a progressão da doença gera complicações como, por exemplo, enfermidades cardiovasculares, uma das principais causas de óbitos nos pacientes dialíticos ${ }^{9}$. Assim sendo, ao total gasto com as sessões de diálise, devem ser somados os valores relativos ao tratamento dessas complicações. Por esse motivo, Best \& Sullivan 10 argumentam que para que a comparação dos custos do transplante renal e da diálise seja válida não devem ser considerados apenas os custos do primeiro ano, mas os custos de longo prazo de ambos os tratamentos. Para esses autores, deve-se ter clareza do ponto a partir do qual o tratamento de transplante renal passa a representar uma economia.

A literatura internacional 11,12,13 evidencia que, apesar dos altos custos iniciais, os custos acumulados do transplante renal são inferiores aos dos tratamentos de diálise na realidade dos Estados Unidos. Eggers 11 reportou que o ponto que equaliza os custos do tratamento de diálise e do transplante renal de doador vivo para o Medicare, calculados com dados de 1979, é de 3 anos e 3 meses, e de 4 anos e 7 meses entre o tratamento de diálise e o transplante renal de doador falecido. Dez anos mais tarde, após a introdução do medicamento imunossupressor ciclosporina, Eggers (1992, apud Loubeau et al. 12), ao analisar informações de 1989, reportou que o ponto de equilíbrio para o Medicare era de 3 anos e 7 meses para transplante de doador vivo e de 4 anos para o transplante de doador falecido.

Schweitzer et al. 13 analisaram os custos incorridos entre 1996 e 1998 pelo Institute for Transplantation nos Estados Unidos, com transplante renal de doador vivo, comparando-os com os custos incorridos com o tratamento de hemodiálise. O ponto de equilíbrio calculado pelos autores foi de 2 anos e 7 meses após o transplante, representando uma economia de mais de US\$ 50.000,00 por paciente em cinco anos após a realização do transplanterenal.Posteriormente,Loubeauetal. ${ }^{11}$ replicaram o mesmo método de cálculo para comparar os custos de hemodiálise e transplante renal incorridos pelo Medicare, em 1998, em oito centros de transplantes localizados em Nova 
York, Estados Unidos. Esses autores encontraram o ponto de equilíbrio de 2 anos e 10 meses. Verifica-se que essas duas pesquisas conduzidas em 1998 demonstraram um encolhimento nos pontos de equilíbrio: cerca de um ano antes daqueles calculados na década anterior.

Apesar da importância de se acompanhar os pontos de equilíbrio para quantificar a economia financeira gerada pelas decisões de investimento público, não foram localizadas publicações que calculem o ponto de equilíbrio na conjuntura brasileira. Realizou-se uma revisão de literatura sobre a comparação dos custos no longo prazo entre o transplante renal e as terapias renais substitutivas. Foi feito o levantamento de artigos originais e de revisão, escritos em inglês, espanhol ou português, indexados nas bases SciELO, PubMed e ISI Web of Knowledge. Também foi realizada busca na Biblioteca Digital Brasileira de Teses e Dissertações. Foram utilizadas as seguintes palavras-chave para a pesquisa (Medical Subject Heading Terms - MeSH): "cost", "health expenditure", "renal dialysis", "renal replacement therapy" e "kidney transplantation". Não houve recorte temporal.

O estudo dos custos envolvidos na assistência prestada fornece informação sobre o impacto da doença, subsidiando a tomada de decisão em relação à alocação de recursos 14. Diante deste contexto, esta pesquisa objetiva analisar o problema dos transplantes renais sob a perspectiva econômica do ponto de vista do principal financiador dos tratamentos da insuficiência renal crônica e comparar os custos arcados pelo SUS no tratamento de terapias renais substitutivas, considerando as modalidades hemodiálise e diálise peritoneal, e transplante renal de doador vivo e falecido no Brasil. E, com base nesta comparação, pretende-se calcular o ponto de equilíbrio e quantificar a economia gerada pelo transplante.

\section{Método de pesquisa}

Letsios 15 classifica os custos em quatro categorias: (a) custos médicos diretos - incluem custos de pessoal, honorários médicos ou de salários, diárias hospitalares, exames complementares e medicamentos; (b) custos diretos não médicos - são os custos com as instalações, construções, transporte dos pacientes e overhead; (c) custos indiretos - são as perdas de produtividade para pacientes e seus familiares e responsáveis; e (d) custos intangíveis - são os custos associados com a dor, o sofrimento e prejuízo na qualidade de vida, bem como o valor do prolongamento da vida. Para proceder ao cálculo de comparação entre os custos do transplante renal e das terapias renais substitutivas, esta pesquisa limita-se aos itens de custo médicos diretos pagos pelo SUS.

Os custos dos tratamentos foram comparados por meio do cálculo do ponto de equilíbrio (ou do inglês break-even point). No estudo microeconômico das empresas, o ponto de equilíbrio é o ponto em que o total das receitas se iguala ao total dos custos e, portanto, o resultado, ou lucro final, é zero 16. Esse conceito foi adaptado para a comparação da evolução de custo entre a terapia renal substitutiva e o transplante renal por diversos autores $11,12,13$, sendo o ponto de equilíbrio aquele em que os custos acumulados do transplante renal equalizam os custos acumulados da terpaia renal substitutiva.

Neste artigo, foi aplicado o método de Schweitzer et al. 13 e Loubeau et al. 12 adaptado à dinâmica de transferência de recursos do SUS para os provedores de saúde para proceder ao cálculo do ponto de equilíbrio. O SUS adota em grande parte o Sistema de Pagamento Retrospectivo, em que as despesas incorridas pelo hospital são ressarcidas a posteriori mediante tabelas de pagamento que determinam os valores repassados em cada procedimento 17 .

As seções a seguir apresentam como as informações de custo foram levantadas e organizadas para efetuar os cálculos.

\section{Custos das terapias renais substitutivas}

Os valores relativos ao custo individual com as terapias renais substitutivas foram extraídos da pesquisa de Cherchiglia et al. 18. Esses autores analisaram os custos ambulatoriais totais, incluindo procedimentos, medicamentos e exames, arcados pelo SUS, no período de 2000-2004, com pacientes que iniciaram hemodiálise e diálise peritoneal no ano de 2000, no Brasil.

Os gastos médios individuais publicados por Cherchiglia et al. 18 foram atualizados para outubro de 2013 pelo deflator calculado com base no Índice Nacional de Preços ao Consumidor Amplo (IPCA/IBGE) 19. Os valores individuais anuais da diálise peritoneal e da hemodiálise após a atualização foram de $\mathrm{R} \$ 44.026,40$ e $\mathrm{R} \$ 34.849,36$ por paciente, respectivamente.

\section{Custos do transplante renal de doador falecido}

Da mesma forma que Schweitzer et al. 13 e de Loubeau et al. ${ }^{12}$, esta pesquisa também divide os custos com transplante renal em custos do primeiro ano e custos dos anos subsequentes. 


\section{- Custos do primeiro ano}

Foram considerados como custos do primeiro ano aqueles referentes à captação do órgão, procedimentos pré-cirúrgicos, procedimentos cirúrgicos, medicamentos imunossupressores, acompanhamentos clínicos, exames e readmissões.

Os itens que compõem os custos relativos à captação do órgão, aos procedimentos pré-cirúrgicos e aos procedimentos cirúrgicos foram levantados em entrevistas realizadas no Hospital Adventista Silvestre, no Rio de Janeiro. Entre outubro e dezembro de 2013 foram realizadas quatro entrevistas para coletar os códigos de faturamento do SUS, duas com um médico nefrologista cirurgião do hospital e duas com um funcionário do setor de Gestão de Custos. Tais profissionais foram selecionados para participar da pesquisa pela experiência e conhecimento em relação ao processo sob análise. $\mathrm{O}$ acesso ao hospital e aos entrevistados foi formalmente autorizado pelo diretor da instituição. Os entrevistados foram esclarecidos sobre a finalidade do estudo e participaram de forma livre da pesquisa, conforme consta no Termo de Consentimento Livre e Esclarecido assinado por ambos.

Os custos de captação do órgão para a cirurgia de doador falecido são compostos por nove itens, cujos códigos de cobrança são: coordenação de sala cirúrgica para a retirada de órgãos e tecidos para transplante (05.03.04.001-0); diária de unidade de terapia intensiva de provável doador de órgãos (05.03.04.004-5); entrevista familiar para doação de órgãos de doadores em morte encefálica (05.03.04.005-3); captação de órgão efetivamente transplantado (05.03.04.008-8); manutenção hemodinâmica de possível doador e taxa de sala para retirada de órgãos (05.03.03.001-5); retirada uni/bilateral de rim (para transplante) (05.03.03.008-2); tipagem sanguínea ABO e outros exames hematológicos em possível doador de órgãos (05.01.07.003-6); avaliação do possível doador falecido de órgãos (05.06.01.005-8); sorologia de possível doador de órgão ou tecido (05.01.07.002-8). A página de internet do Sistema de Gerenciamento da Tabela de Procedimentos e Órtese, Prótese e Materiais Especiais do SUS disponibiliza mais informações sobre cada código (SIGTAP. http://sigtap.datasus.gov.br, acessado em 04/Fev/2014).

Os custos relativos aos procedimentos précirúrgicos do transplante de doador falecido são compostos por dois itens, que possuem os seguintes códigos: acompanhamento de pacientes no pré-transplante de órgãos (05.06.01.0040 ); exames para inclusão em lista de candidatos a transplante de pâncreas, pulmão ou rim (05.01.07.006-0). Para os procedimentos cirúrgi- cos de transplante de doador falecido foi utilizado o código do transplante de rim (órgão de doador falecido) (05.05.02.009-2).

Os valores dos acompanhamentos clínicos e exames, medicamentos imunossupressores e readmissões foram extraídos da pesquisa de Guerra Júnior et al. 8. Esses autores analisaram uma coorte histórica composta por todos os pacientes que realizaram transplante renal de doador vivo ou falecido no Brasil, em todos os centros transplantadores do SUS, no período de janeiro de 2000 a dezembro de 2003, e que fizeram uso dos medicamentos imunossupressores ciclosporina ou tacrolimus.

Guerra Júnior et al. 8 apresentam dois conjuntos desses itens de custo - um referente ao esquema terapêutico da ciclosporina e outro referente ao esquema terapêutico do tacrolimus. No presente estudo, foram adotados os valores relacionados à ciclosporina, pois de acordo com as conclusões da pesquisa de Saturnino 20 e de Guerra Júnior 21, a ciclosporina é o esquema terapêutico mais custo efetivo para o SUS.

\section{- Custos dos anos subsequentes}

Os itens que compõem os custos dos anos subsequentes são: medicamentos imunossupressores, custos de acompanhamento e exames e custos líquidos de falha de enxerto. Guerra Júnior et al. ${ }^{8}$ publicaram dados do primeiro ano após o transplante renal e dos três anos subsequentes, que corresponderam ao período de acompanhamento da coorte. Nos três anos subsequentes, foram utilizados os custos referentes ao esquema terapêutico da ciclosporina para compor o item de custo dos medicamentos imunossupressores. Os valores dos acompanhamentos clínicos e exames foram também extraídos da pesquisa de Guerra Júnior et al. 8. Os custos líquidos de falha de enxerto, por sua vez, foram calculados com a fórmula de Schweitzer et al. 13 e Loubeau et al. 12, qual seja: (custo pago pelo SUS com a hemodiálise - custo com medicamentos imunossupressores) x $0,73 \%$ a probabilidade mensal que $o$ paciente sofra falha de enxerto.

\section{Custos do transplante renal de doador vivo}

\section{- Custos do primeiro ano}

Foram considerados como custos do primeiro ano aqueles referentes a procedimentos pré-cirúrgicos, procedimentos cirúrgicos, medicamentos imunossupressores, acompanhamentos clínicos, exames e readmissões. Os custos referentes à captação do órgão não foram considerados por se tratar de uma cirurgia de doador vivo. 
Os custos dos procedimentos pré-cirúrgicos do transplante de doador vivo são compostos por cinco itens cujos códigos são: acompanhamento de pacientes no pré-transplante de órgãos (05.06.01.004-0); tipagem sanguínea ABO e outros exames hematológicos em possível doador de órgãos (05.01.07.003-6); sorologia de possível doador de órgão ou tecido (05.01.07.002-8); exames para investigação clínica no doador vivo de rim, fígado ou pulmão (1 1 a fase) (05.01.07.0087); exames para investigação clínica no doador vivo de rim (complementação da 1a fase) (05.01.07.010-9).

Para os procedimentos cirúrgicos de transplante de doador vivo foram utilizados os código do transplante de rim (órgão de doador vivo - código 05.05.02.010-6) e nefroureterectomia unilateral para transplante (cirurgia para transplante doador vivo - código 05.03.02.0028).

Os valores dos acompanhamentos clínicos e exames, medicamentos imunossupressores e readmissões foram extraídos de Guerra Júnior et al. ${ }^{8}$.

\section{- Custos dos anos subsequentes}

Os itens de custos dos anos subsequentes computados também são os mesmos dos custos do transplante renal de doador falecido: medicamentos imunossupressores, custos de acompanhamento e os custos líquidos de falha de enxerto. Procedeu-se aos mesmos cálculos descritos na seção Custos dos Anos Subsequentes dos doadores falecidos.

Os valores extraídos de Guerra Júnior et al. 8 foram atualizados para outubro de $2013 \mathrm{com}$ base no IPCA/IBGE 19, de modo que todas as quantias estejam equalizadas no período base. Com isso, computaram-se, primeiramente, os gastos marginais mensais e, em seguida, os gastos mensais acumulados. As informações dos quatro anos de que se tem informação de custo da coorte foram estruturadas no software Microsoft Excel 2007 (Microsoft Corp., Estados Unidos).

\section{Resultados}

O ponto de equilíbrio entre a hemodiálise e o transplante renal de doador falecido

O comportamento dos custos acumulados no longo prazo do transplante renal de doador falecido e da hemodiálise está apresentado na Figura 1. O custo acumulado da hemodiálise é representado por uma reta de inclinação positiva, o que indica que são acrescidos valores incrementais mensais constantes de $\mathrm{R} \$ 2.904,03$, que corresponde R\$ 34.849,36 (calculado na seção Custos das Terapias Renais Substitutivas) rateados por 12 .

Com relação aos custos do transplante renal, verifica-se que a reta se inicia em um patamar superior à reta de custo da hemodiálise: $\mathrm{R} \$$ 36.781,06. O tratamento apresenta custos mais elevados no início, o que se deve aos procedimentos pré-cirúrgicos e cirúrgicos. Os custos com medicamentos imunossupressores também são mais elevados nos estágios iniciais do tratamento. No primeiro ano, são gastos um total de $\mathrm{R}$ \$ 78.401,26. No mês 13 há uma redução dos valores incrementais e consequente mudança no comportamento da reta. No segundo, terceiro e quarto ano, passam a ser acrescidos $\mathrm{R} \$ 8.473,72$, $\mathrm{R} \$ 7.102,92$ e R\$ 8.335,91, respectivamente. Esse comportamento vai de encontro com o descrito na literatura 11,12,13 que prevê valores decrescentes no longo prazo.

O ponto de equilíbrio que equaliza os custos do transplante renal de doador falecido e da hemodiálise, sob a perspectiva do SUS, ocorre no mês 32. Isso significa dizer que os custos acumulados da hemodiálise são compensados pelos custos do transplante renal dois anos e oito meses após a cirurgia de transplante renal. Ao final dos quatro anos de acompanhamento da coorte é alcançada uma economia de R\$ $37.083,63$ por paciente.

Considerando-se a situação de que todo o contingente de 100 mil brasileiros que, em 2013, estavam sendo submetidos à terapia renal substitutiva recebesse um transplante renal e a taxa de crescimento de 20 mil pacientes/ano, conforme estimativas da SBN 5, a opção pelo transplante renal de doador falecido poderia significar para os cofres públicos uma economia de $\mathrm{R} \$ 5,9$ bilhões em quatro anos.

\section{O ponto de equilíbrio entre a hemodiálise e o transplante renal de doador vivo}

Os custos acumulados do transplante renal de doador vivo e da hemodiálise foram plotados em um gráfico e estão apresentados na Figura 2. Observa-se que a curva dos custos acumulados do transplante renal de doador vivo possui comportamento semelhante à curva dos custos acumulados do transplante renal de doador falecido. A reta se inicia no valor $\mathrm{R} \$ 28.263,82$, patamar superior ao custo da hemodiálise. Os elevados custos iniciais devem-se também aos procedimentos pré-cirúrgicos, cirúrgicos e medicamentos imunossupressores.

No primeiro ano, são gastos um total de $\mathrm{R} \$$ 69.884,02. No segundo, terceiro e quarto ano, passam a ser acrescidos $\mathrm{R} \$ 8.473,32, \mathrm{R} \$ 7.102,92 \mathrm{e}$ 
Figura 1

Custos acumulados por paciente do transplante renal de doador falecido e da hemodiálise.

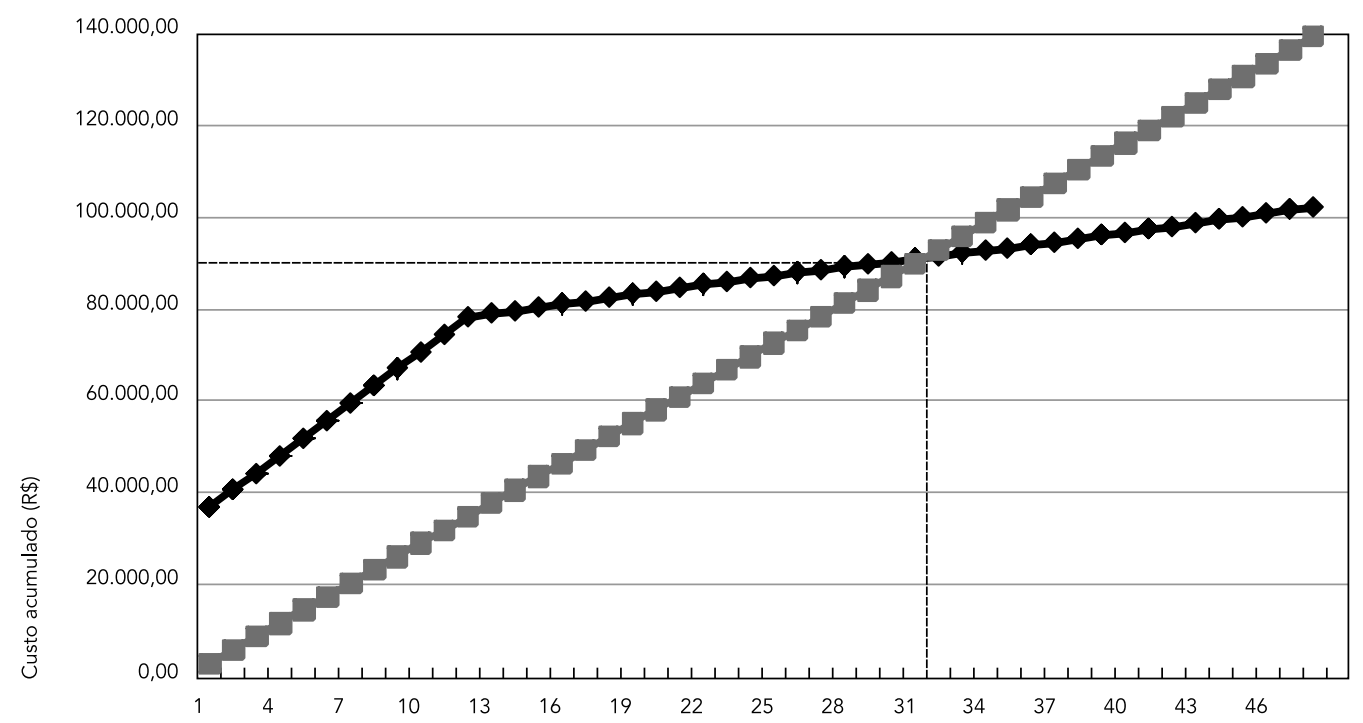
Transplante renal (doador falecido)

- Hemodiálise

Figura 2

Custos acumulados por paciente do transplante renal de doador vivo e da hemodiálise.

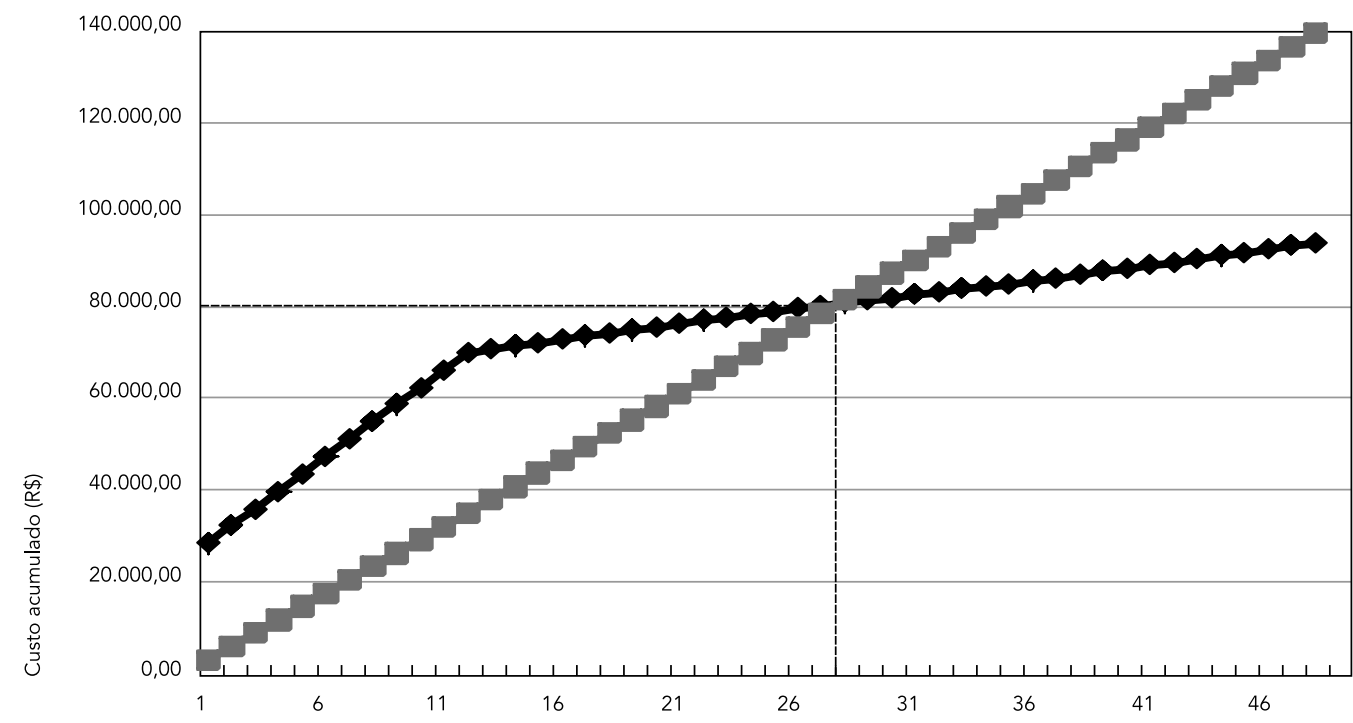
$\neg$ Transplante renal (doador vivo)
$\rightarrow$ Hemodiálise


$\mathrm{R} \$$ 8.335,91, respectivamente. Com isso, da mesma forma que o encontrado na análise anterior, os valores incrementais mensais da hemodiálise são superiores aos valores incrementais mensais do transplante renal de doador vivo, sobretudo após o mês 13. Como consequência, os custos acumulados do transplante renal de doador vivo são também superados pelos custos acumulados da hemodiálise.

O ponto de equilíbrio que equaliza os custos do transplante renal de doador vivo e da hemodiálise, sob a perspectiva do SUS, dá-se no vigésimo oitavo mês, ou seja, os custos acumulados da hemodiálise são compensados pelos custos do transplante renal de doador vivo dois anos e quatro meses após a cirurgia de transplante. Segundo os cálculos realizados, ao final dos quatro anos de acompanhamento da coorte, é alcançada uma economia de $\mathrm{R} \$ 45.600,87$ por paciente. Isso significaria, valendo-se do mesmo exercício realizado na seção anterior, uma economia de recursos públicos de $\mathrm{R} \$ 7,3$ bilhões em quatro anos, levando-se em consideração o contingente da população brasileira que necessita de terapia renal substitutiva, conforme dados da SBN 5 .

O ponto de equilíbrio entre a diálise peritoneal e o transplante renal de doador falecido

A Figura 3 apresenta o gráfico dos custos acumulados no longo prazo do transplante renal de doador falecido e da diálise peritoneal. Verifica-se que a curva dos custos acumulados do tratamento de diálise peritoneal possui comportamento semelhante à curva dos custos acumulados do tratamento de hemodiálise. O custo acumulado da diálise peritoneal é representado por uma reta de inclinação positiva, o que indica que são acrescidos valores incrementais mensais constantes de $\mathrm{R} \$ 3.668,87$, que corresponde a $\mathrm{R} \$$ 44.026,40 rateados por 12 .

De acordo com os dados publicados por Cherchiglia et al. 18, explicitados no item Custos das Terapias Renais Substitutivas, os custos da diálise peritoneal são $26 \%$ superiores aos da hemodiálise. Portanto, os custos acumulados do transplante renal de doador falecido são superados pelos custos acumulados da diálise peritoneal quatro meses antes quando comparados com os custos da hemodiálise.

Dessa forma, o ponto de equilíbrio que equaliza os custos do transplante renal de doador falecido e da diálise peritoneal, sob a perspectiva do SUS, encontra-se no vigésimo quarto mês, ou seja, os custos acumulados da diálise peritoneal são compensados pelos custos do transplante renal dois anos após a cirurgia de transplante renal de doador falecido. E, ao final dos quatro anos de acompanhamento da coorte, é alcançada uma economia financeira para o SUS de R $\$ 73.791,80$ por paciente, totalizando R\$11,8 bilhões de economia em quatro anos, caso o transplante renal de doador falecido seja o tratamento adotado para a população brasileira que depende de terapia renal substitutiva, conforme dados da SBN 5 .

O ponto de equilíbrio entre a diálise peritoneal e o transplante renal de doador vivo

A Figura 4 apresenta o gráfico dos custos no longo prazo do transplante renal de doador vivo e da diálise peritoneal.

O ponto de equilíbrio que equaliza os custos do transplante renal de doador vivo e da diálise peritoneal, sob a perspectiva do SUS, encontrase no vigésimo primeiro mês, ou seja, um ano e nove meses após a cirurgia de transplante renal de doador vivo. De acordo com os cálculos elaborados neste estudo, ao final dos quatro anos, é alcançada uma economia de $\mathrm{R} \$$ 82.309,04 por paciente, vale dizer, a opção pelo transplante renal de doador vivo, relativamente ao tratamento por diálise peritoneal, significaria para os cofres públicos, em quatro anos, uma economia de R\$ 13,2 bilhões, tendo em vista o número de brasileiros que padece de insuficiência renal crônica e que depende de terapia renal substitutiva para sobreviver, conforme dados da SBN 5.

A Tabela 1 sintetiza os pontos de equilíbrio e as economias alcançadas em quatro anos ao comparar os custos do transplante renal e das diálises no Brasil.

\section{Discussão}

Os cálculos realizados indicam que, no longo prazo, os custos médicos diretos acumulados das terapias renais substitutivas superam os custos acumulados do transplante renal de doador vivo e de doador falecido e que os custos associados ao transplante renal são compensados em um período inferior a três anos. Significa dizer que o transplante renal pode gerar para o SUS uma economia financeira em relação às diálises.

O ponto de equilíbrio sob a perspectiva do SUS é anterior ao calculado sob a perspectiva do Medicare, mesmo nos estudos mais recentes, que analisam dados de 1996-1998. Assim, o tempo para que o alto custo inicial do transplante renal seja recuperado pelos cofres públicos brasileiros é menor do que nos Estados Unidos.

Os custos que se compensam mais rapidamente no contexto brasileiro são os da diálise 


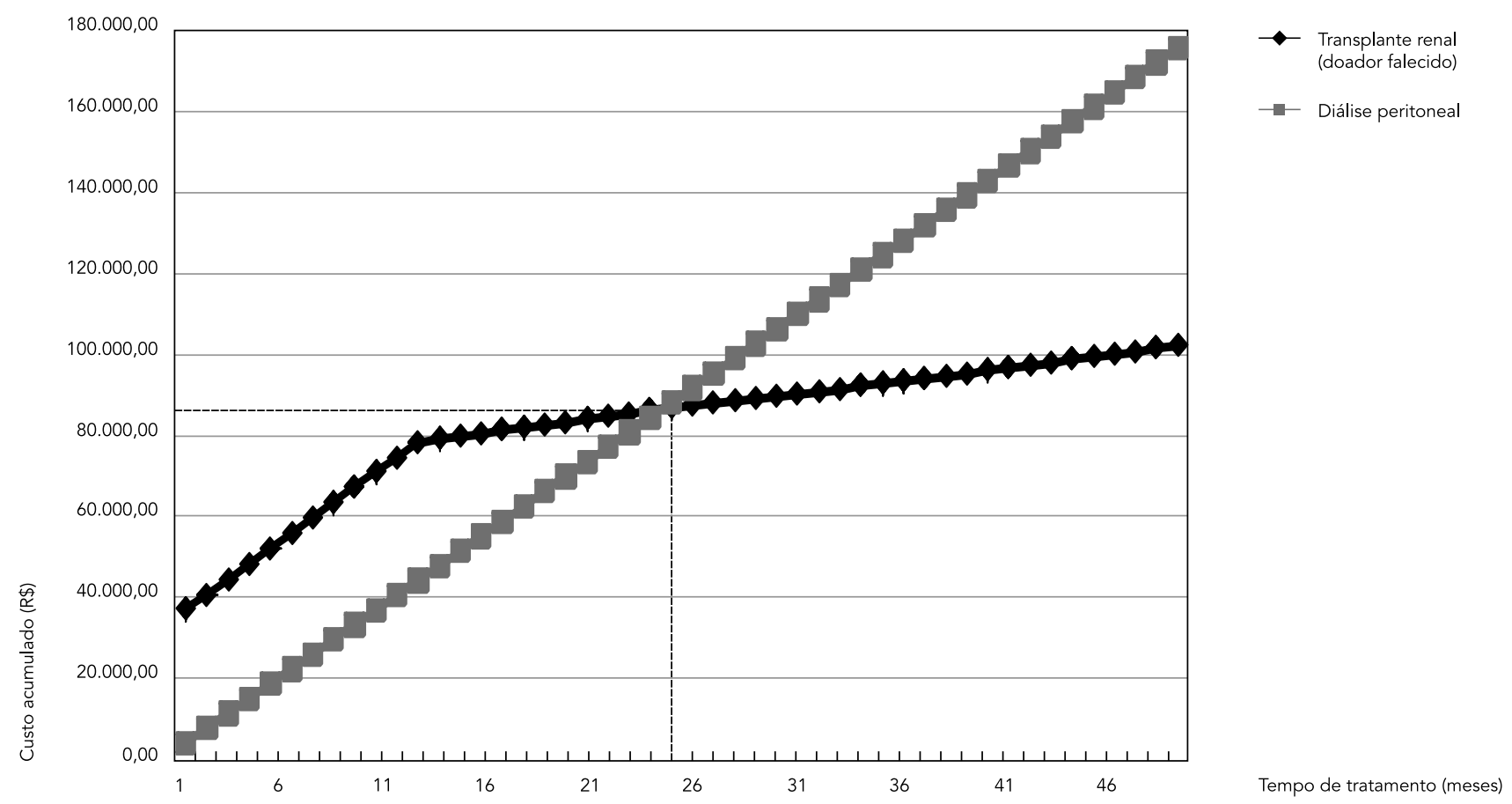

peritoneal relativamente aos do transplante renal de doador vivo, cujo ponto de equilíbrio ocorre em um ano e nove meses. Já os custos de hemodiálise, relativamente aos de transplante renal de doador falecido, são os que levam mais tempo para se compensar no Brasil: dois anos e oito meses. Levando-se em consideração apenas os custos médicos diretos, assim como o contingente de 100 mil brasileiros que, em 2013, estavam sendo submetidos à terapia renal substitutiva, e a taxa de crescimento de $20 \mathrm{mil}$ pacientes/ano, conforme estimativas da SBN 5, a opção pelo transplante renal, relativamente às terapia renal substitutiva, geraria uma economia de recursos públicos bastante significativa, variando de $\mathrm{R} \$ 5,9$ bilhões a R \$ 13,2 bilhões nos quatro anos analisados neste estudo, conforme sintetizado na Tabela 1.

O método de comparação dos custos do transplante renal e das diálises é também uma importante contribuição deste estudo. O método utilizado na literatura internacional foi adaptado para o contexto brasileiro e se encontra aderente à dinâmica da transferência de recursos do SUS para os provedores de saúde. O mesmo foi ex- posto de forma detalhada a fim de ser facilmente replicado na condução de estudos futuros.

Os custos para o SUS relacionados à insuficiência renal crônica são constantemente afetados pela tecnologia, inovações nos medicamentos imunossupressores, itens médicos que passam a ser financiados pelo governo etc. Saturnino 22 evidencia a importância de se avaliar a introdução de novos medicamentos de manutenção do transplante renal dado o papel social do SUS ao arcar em sua integralidade com o tratamento do transplante. Em razão disso, a lógica de incorporação de novos fármacos, aliada a maiores custos diante dos recursos finitos da saúde, vem sendo objeto de discussão para a incorporação de novas tecnologias dentro do Ministério da Saúde.

Os resultados do transplante têm sido tema de estudos e melhorias ao longo do tempo. Schweitzer et al. 13 apresentaram que eficiências nas operações dos hospitais podem também trazer redução de custos em pacientes transplantados. Assim como o transplante renal, inovações relacionadas às diálises podem também trazer alterações nos custos dessa intervenção. 


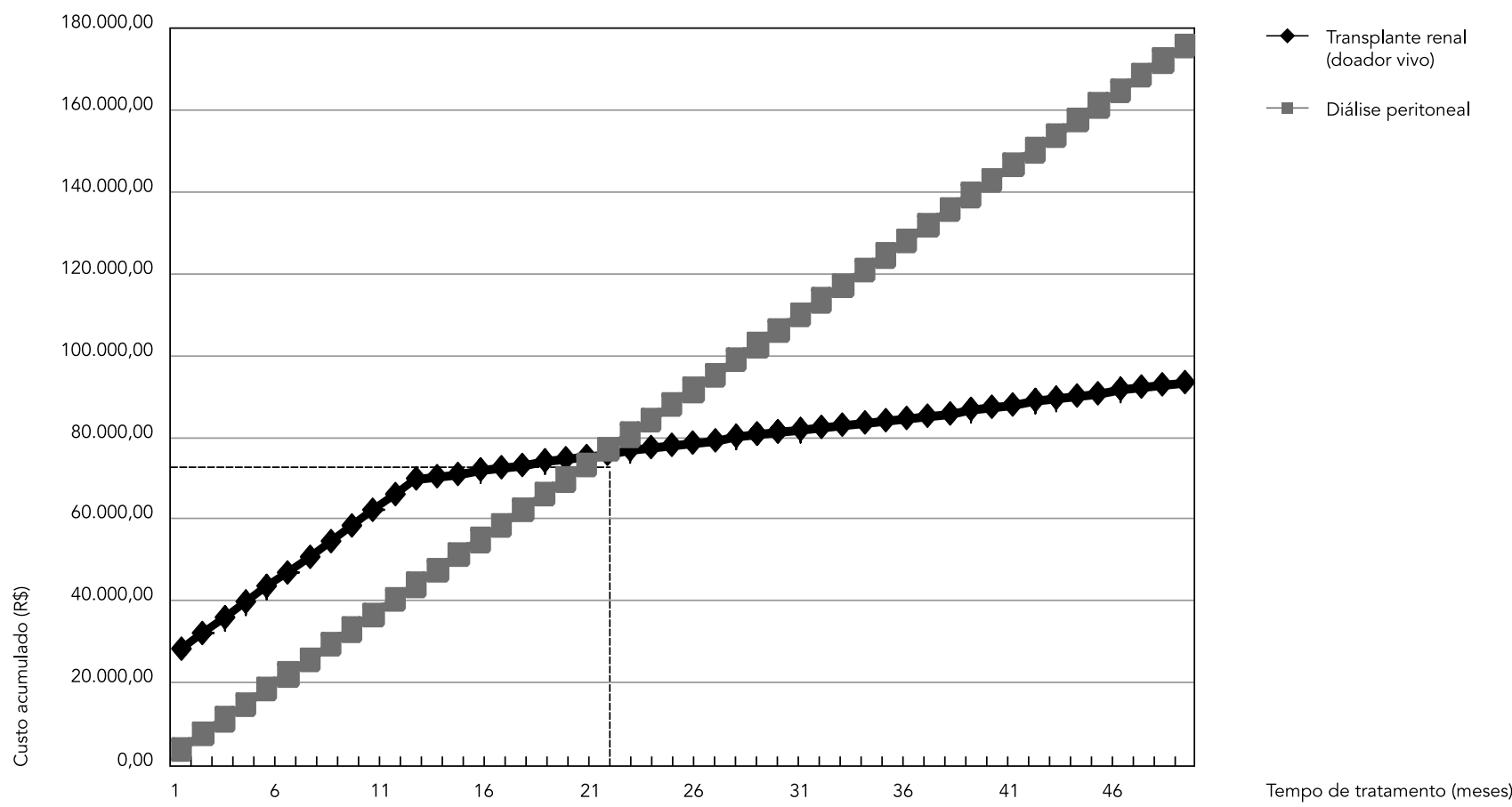

Tabela 1

O ponto de equilíbrio e economia alcançada por paciente em 4 anos, ao comparar os custos do transplante renal e das diálises no Brasil.

Intervenções analisadas
Ponto de equilíbrio

Economia para os cofres públicos em quatro anos (R\$)

Por paciente

$\begin{array}{ccc} & \text { Por paciente } & \text { Economia total * } \\ 2 \text { anos e } 8 \text { meses } & 37.083,63 & 5,9 \text { bilhões } \\ 2 \text { anos e } 4 \text { meses } & 45.600,87 & 7,3 \text { bilhões } \\ 2 \text { anos } & 73.791,80 & 11,8 \text { bilhões } \\ 1 \text { ano e } 9 \text { meses } & 82.309,04 & 13,2 \text { bilhões }\end{array}$

Economia total *
Hemodiálise (transplante renal de doador falecido)

Hemodiálise (transplante renal de doador vivo)

Diálise peritoneal (transplante renal de doador falecido)

Diálise peritoneal (transplante renal de doador falecido)

* Considerando-se o contingente de 100 mil brasileiros que, em 2013, estavam sendo submetidos a terapias renais substitutivas e a taxa de crescimento de 20 mil pacientes/ano, conforme estimativas da Sociedade Brasileira de Nefrologia 5.

Finalmente, espera-se que os gastos alocados no tratamento dessa enfermidade continuem a aumentar em virtude do envelhecimento da população e do aumento da incidência de patologias cujo avanço de sua história natural evolui para a falência renal crônica, como o diabetes e a hipertensão, principais causas da insuficiência renal crônica 5 .
Esses argumentos elucidam a importância de um método que permita a comparação dos custos de ambas as intervenções e que possa ser facilmente replicado posteriormente. As informações contidas neste estudo explicitam a realidade de custo para o SUS, convergentes com resultados internacionais de que o transplante renal apresenta menores gastos para o financiador. 
Além dos benefícios financeiros, estudos anteriores 22,23,24,25 comprovaram que o transplante renal é a alternativa mais efetiva dentre as modalidades de tratamento da insuficiência renal crônica, pois permite, na maioria dos casos, a reintegração do paciente às suas atividades cotidianas, aumentando a probabilidade de uma melhor expectativa e qualidade de vida em relação aos pacientes submetidos às diálises.

No entanto, apesar de o Brasil ser um dos países que mais realizam transplantes no mundo, possui uma das menores taxas de doação - cerca de cinco doadores por milhão de habitantes 26 - e o problema da escassez da oferta de órgãos vem gerando uma rápida expansão na lista de espera por transplante 27. Marinho 28 verifica que a fila de espera por um rim é maior quando comparada aos demais órgãos: o tempo médio de espera na fila do transplante de rim ajustado pela mortalidade é de 5,5 anos; e sem essa estimativa, o tempo aumenta para aproximadamente 11,1 anos.

Marinho 7 relata os impactos negativos que as filas exercem nos pacientes no que tange ao bem-estar, as probabilidades de cura, a extensão das sequelas, os custos gerados pela demora de atendimento e pela manutenção dos pacientes na fila. A falta de doações efetivas leva o sistema de transplantes renais no Brasil a executar uma quantidade de cirurgias abaixo das necessidades da população 2,27 , gerando um descompasso entre a oferta e a demanda de rim no país e as longas esperas.

A negativa familiar é um dos problemas que limita a oferta de órgãos no Brasil. A recusa se deve à falta de explicação à família por parte do médico do que é a morte cerebral; ao medo da comercialização de órgãos e assistência médica julgada inadequada 29; ao desconhecimento da vontade prévia do potencial doador; a fatores religiosos, sociais e culturais; ao desconhecimento familiar do sistema de alocação; à entrevista inadequada com a equipe do SUS; ao atendimento insatisfatório durante a internação; e às dificuldades com a equipe que acompanhou o paciente 27 .

O funcionamento adequado do processo de captação de órgãos é fundamental para garantir a efetividade da doação. Contudo, falhas em aspectos relacionados à logística, tais como falta de leitos de unidades de terapia intensiva (UTI) ou equipamentos para diagnóstico de morte encefálica e/ou falta de neurologista ou neurocirurgião 26 têm se caracterizado como um limitador. Nesse sentido, a estrutura de incentivos, com a qual as instituições envolvidas se deparam, constitui-se um fator decisivo no desempenho do sistema de transplante 27 .
Os processos de gestão não se encontram no mesmo nível de maturidade em todos os estados brasileiros: existem disparidades significativas em relação à captação e à quantidade de transplante renal realizada entre as unidades da federação 30,31,32. Pestana et al. 32 descrevem os avanços de gestão do Estado de São Paulo que permitiram o estado alcançar taxas de doação superiores à média nacional e próximo da média de países com os melhores desempenhos atuais. De fato, existe elevada "capacidade ociosa", no Brasil, de cada 8 potenciais doadores, apenas 1 é notificado e somente $20 \%$ deles são utilizados como doadores de múltiplos órgãos 4,33.

Entretanto, ampliar na totalidade o programa de transplante renal exige que sejam apurados os demais gastos públicos além dos serviços médicos, incluindo, por exemplo, custos de instalações, planejamento e programas de formação, a fim de assegurar melhor estimativa do custo global 14 . Idealmente, tais custos deveriam ser mensurados e comparados para ambos os programas, com a realização de exercícios regionalizados e consideradas as diferenças de sexo, idade e presença de comorbidades, a fim de verificar os resultados diferenciados no território nacional.

Além disso, há variáveis qualitativas importantes que impactariam os exercícios de mensuração dos ganhos financeiros expostos na seção precedente. Nem todo o contingente de pacientes com insuficiência renal crônica está apto à realização do transplante e, portanto, cada perfil deve ser analisado 34. Em sua maioria, os pacientes submetidos à diálise peritoneal são aqueles não elegíveis à hemodiálise, por apresentarem comorbidades e condições físicas mais graves. Consequentemente, esses pacientes são menos transplantados 22. Dessa maneira, o potencial de ganho financeiro quantificado anteriormente pode não ser alcançado na totalidade.

\section{Considerações finais}

Ao estimar o potencial de economia gerada no longo prazo pelo transplante renal, este artigo gera um incentivo à promoção de ações que aumentem a captação de órgãos. Essas ações podem ser de ordem gerencial, por meio da melhoria da logística e do processo de captação do órgão, ou mesmo programas de conscientização da população quanto à relevância do tema, visando à redução da negativa familiar. Este estudo, por conseguinte, é útil na investigação da conjuntura atual e na formulação de políticas públicas relacionadas aos transplantes de órgãos no Brasil. Medidas financeiras aliadas a análises de sobrevida e qualidade de vida são capazes de 
gerar importantes informações para os serviços públicos de saúde e cooperar com o uso eficiente de recursos limitados.

A análise realizada na seção anterior permite identificar oportunidades de aprofundamento deste estudo em pesquisas futuras, com a incorporação de outros indicadores de caráter qualitativo e cômputo dos custos diretos não médicos.

\section{Colaboradores}

S. B. Silva contribuiu na concepção e projeto da pesquisa, na análise e interpretação dos dados e na redação do artigo. H. M. Caulliraux, C. A. S. Araújo e E. Rocha colaboraram na orientação na concepção e projeto da pesquisa, na orientação, análise e interpretação dos dados, e revisão crítica relevante do conteúdo intelectual do artigo.

\section{Referências}

1. Bastos MG, Kirsztajn GM. Doença renal crônica: importância do diagnóstico precoce, encaminhamento imediato e abordagem interdisciplinar estruturada para melhora do desfecho em pacientes ainda não submetidos à diálise. J Bras Nefrol 2011; 33:93-108.

2. Garcia GG, Harden P, Chapman J. O papel global do transplante renal. J Bras Nefrol 2012; 34:1-7.

3. Godoy MR, Balbinotto Neto G, Ribeiro EP. Earnings and chronic renal disease. Transplantat Proc 2010; 39:378-80.

\section{Agradecimentos}

Os autores agradecem ao Hospital Adventista Silvestre pelo apoio dado à pesquisa e pela valorosa contribuição das pessoas entrevistadas.
4. Marinho A, Cardoso SS, Almeida VV. Os transplantes de órgãos nos estados brasileiros. Rio de Janeiro: Instituto de Pesquisa Econômica Aplicada; 2007. (Texto para Discussão, 1317).

5. Sociedade Brasileira de Nefrologia. Censo de diálise. http://www.sbn.org.br/pdf/censo_2013-14-05. pdf (acessado em 01/Fev/2014).

6. Vecina Neto G, Malik AM. O futuro dos serviços de saúde no Brasil. In: Vecina Neto G, Malik AM, organizadores. Gestão em saúde. Rio de Janeiro: Editora Guanabara Koogan; 2012. p. 351-7. 
7. Marinho A. Um estudo sobre as filas para internações e para transplantes no sistema único de saúde brasileiro. Rio de Janeiro: Instituto de Pesquisa Econômica Aplicada; 2004. (Texto para Discussão, 1055).

8. Guerra Júnior AA, Acúrcio FA, Andrade EIG, Cherchiglia ML, Cesar CC, Queiroz OV, et al. Ciclosporina versus tacrolimus no transplante renal no Brasil: uma comparação de custos. Cad Saúde Pública 2010; 26:163-74.

9. Peres LAB, Matsuo T, Ann HK, Camargo MTA, Rohde NRS, Uscocovich VSM, et al. Causas de óbitos em pacientes renais crônicos em programa dialítico. Rev Bras Clín Méd 2010; 8:495-9.

10. Best JH, Sullivan SD. The changing cost-effectiveness of renal transplantation: the impact of improvements in immunossupressive therapy. Transplant Rev 1998; 12:34-41.

11. Eggers PW. Analyzing the cost effectiveness of kidney transplantation. In: Proceedings of the 19th National Meeting of the Public Health Conference on Records and Statistics. Washington DC: U.S. Department of Health and Human Services; 1984. p. 216-9.

12. Loubeau PR, Loubeau J, Jantzen R. The economics of kidney transplantation versus hemodialysis. Prog Transplant 2001; 11:291-7.

13. Schweitzer EJ, Wiland A, Evans D, Novak M, Connerny I, Norris L, et al. The shrinking renal replacement therapy "break-even" point. Transplant 1998; 66:1702-8.

14. Oliveira ML, Santos LMP, Silva EN. Bases metodológicas para estudos de custos da doença no Brasil. Rev Nutr 2014; 27:585-95.

15. Letsios A. The effect of the expenditure increase in the morbidity and the mortality of patients with end stage renal disease: the USA case. Hippokratia 2011; 15:16-21.

16. Levine D, Boldrin M. Against intellectual monopoly. Cambridge: Cambridge University Press; 2008.

17. Médici AC. Sistemas de financiamento e gestão hospitalar: uma aplicação ao caso brasileiro. In: Vecina Neto G, Malik AM, organizadores. Gestão em saúde. Rio de Janeiro: Editora Guanabara Koogan; 2012. p. 50-72.

18. Cherchiglia ML, Gomes IC, Alvares J, Guerra Júnior A, Acurcio FA, Andrade EIG, et al. Determinantes dos gastos com diálises no Sistema Único de Saúde, Brasil, 2000 a 2004. Cad Saúde Pública 2010; 26:1627-41.

19. Instituto Brasileiro de Geografia e Estatística. Índice Nacional de Preços ao Consumidor Amplo IPCA e Índice Nacional de Preços ao Consumidor - INPC. http://www.ibge.gov.br/home/estatistica/ indicadores/precos/inpc_ipca/defaultinpc.shtm (acessado em 20/Jan/2014).

20. Saturnino LTM. Análise de custo-efetividade dos inibidores da rapamicina para o tratamento de imunossupressão no transplante renal [Tese de Doutorado]. Belo Horizonte: Universidade Federal de Minas Gerais; 2012.
21. Guerra Júnior AA. Avaliação de custo-efetividade entre esquemas imunossupressores utilizados no transplante renal - um estudo farmacoepidemiológico e farmacoeconômico sobre o programa nacional de medicamentos excepcionais no SUS de 2000 a 2004 [Tese de Doutorado]. Belo Horizonte: Universidade Federal de Minas Gerais; 2010.

22. Alvares J. Avaliação da qualidade de vida e análise de custo utilidade das Terapias Renais Substitutivas no Brasil [Tese de Doutorado]. Belo Horizonte: Universidade Federal de Minas Gerais; 2011.

23. Sesso R, Eisenberg JM, Stabile C, Draibe S, Ajzen $\mathrm{H}$, Ramos O. Cost-effectiveness analysis of the treatment of end-stage renal disease in Brazil. Int J Technol Assess Health Care 1990; 6:107-14.

24. Arredondo A, Rangel R, Icaza E. Costo-efectividad de intervenciones para insuficiencia renal crónica terminal. Rev Saúde Pública 1998; 32:556-65.

25. Schnuelle P, Lorenz D, Trede M, van de Woude FJ. Impact of renal cadaveric transplantation on survival in end-stage renal failure: evidence for reduced mortality risk compared with hemodialysis during long-term follow-up. J Am Soc Nephrol 1998; 9:2135-41.

26. Silva EN. Ensaios em economia da saúde: transplantes de rim [Tese de Doutorado]. Porto Alegre: Faculdade de Ciências Econômicas, Universidade Federal do Rio Grande do Sul; 2008.

27. Costa CKF. Ensaios sobre a economia dos transplantes renais no Brasil: incentivos e eficiência [Tese de Doutorado]. Porto Alegre: Faculdade de Ciências Econômicas, Universidade Federal do Rio Grande do Sul; 2012.

28. Marinho A. Um estudo sobre as filas para transplantes no Sistema Único de Saúde brasileiro. Cad Saúde Pública 2006; 22:2229-39.

29. Cunha CB, León ACP, Schramm JMA, Carvalho MS, Souza Junior PRB, Chain R. Tempo até o transplante e sobrevida em pacientes com insuficiência renal crônica no Estado do Rio de Janeiro, Brasil, 1998-2002. Cad Saúde Pública 2007; 23:805-13.

30. Costa CKF, Balbinoto Neto G, Sampaio LMB. Eficiência dos estados brasileiros e do Distrito Federal no sistema público de transplante renal: uma análise usando método DEA (Análise Envoltória de Dados) e índice de Malmquist. Cad Saúde Pública 2014; 30:1667-79.

31. Marinho A, Cardoso SS, Almeida VV. Desigualdade de transplantes de órgãos no Brasil: análise do perfil dos receptores por sexo e raça ou cor. Rio de Janeiro: Instituto de Pesquisa Econômica Aplicada; 2011. (Texto para Discussão, 1629).

32. Pestana JOM, Galante NZ, Tedesco-Silva Jr. H, Harada KM, Garcia VD, Abbud-Filho M, et al. O contexto do transplante renal no Brasil e sua disparidade geográfica. J Bras Nefrol 2011; 33:472-84.

33. Marinho A, Cardoso SS, Almeida VV. Disparidade nas filas para transplantes de órgãos nos estados brasileiros. Cad Saúde Pública 2010; 26:786-96.

34. Torres GV, Mendonça AEO, Amorim IG, Oliveira ICM, Dantas RAN, Freire ILS. Perfil de pacientes em lista de espera para transplante renal. Rev Enferm UFSM 2013; 3:700-8. 


\section{Abstract}

This study aimed to compare the direct medical costs of renal transplantation and renal replacement therapies, specifically hemodialysis and peritoneal dialysis, from the perspective of the Brazilian Unified National Health System (SUS). Renal replacement therapies costs were based on data published in the literature. Cost items for kidney transplant were identified in a private hospital based on procedure codes used for charging the SUS, and other items were taken from the literature. In the four years covered by the study, cadaver kidney transplant generated per-patient savings of BRL 37,000 and BRL 74,000 compared to hemodialysis and peritoneal dialysis, respectively. Savings were even greater with living donor kidney transplant: BRL 46,000 and $B R L$ 82,000 compared to hemodialysis and peritoneal dialysis, respectively. This result, together with survival and quality-of-life analyses, characterizes kidney transplant as the best clinical and financial alternative, thus supporting public policies for organ transplants in Brazil.

Kidney Transplantation; Costs and Cost Analysis; Economics

\section{Resumen}

El objetivo del presente estudio fue comparar los costes médicos directos del trasplante renal y de las terapias renales substitutivas, específicamente la hemodiálisis y la diálisis peritoneal, bajo la perspectiva del Sistema Único de Salud (SUS). Los costes de las terapias renales substitutivas se extrajeron de información publicada en la literatura. Los ítems de coste previstos del trasplante renal se identificaron en un hospital privado, a partir de la recogida de códigos de procedimientos utilizados para el cobro del SUS y los demás ítems extraídos de la literatura. El resultado de esta investigación indica que, en el período de los 4 años cubierto por este estudio, el trasplante renal del donante fallecido genera un ahorro, por paciente, de $R \$ 37$ mil y $R \$ 74$ mil en relación al hemodiálisis y al diálisis peritoneal, respectivamente. En cuanto al trasplante renal del donante vivo, los ahorros son incluso mayores: $R \$ 46$ mil y $R \$ 82$ mil, en relación a la hemodiálisis y a la diálisis peritoneal, respectivamente. Este resultado, junto con análisis de supervivencia y calidad de vida, puede caracterizar el trasplante renal como la mejor alternativa desde el punto de vista financiero y clínico, auxiliando en la formulación de políticas públicas relacionadas con los trasplantes de órganos en Brasil.

Trasplante de Riñón; Costos y Análisis de Costo; Economía
Recebido em 01/Fev/2015

Versão final reapresentada em 05/Out/2015

Aprovado em 18/Fev/2016 